\title{
ITERATIVE SOLUTION OF LINEAR EQUATIONS IN BANACH SPACES
}

\author{
J. WAYNE PACE AND W. G. DOTSON, JR.
}

\begin{abstract}
Several results are obtained which generalize recent results of Kwon and Redheffer concerning the solution of $u-T u=f$ by means of iteration.
\end{abstract}

Throughout this note $E$ is a (real or complex) Banach space and $T$ is a continuous linear operator from $E$ to $E$. Let $f \in E$. In [1] Browder and Petryshyn considered the equation

$$
u-T u=f
$$

and the convergence (to solutions $u$ of (1)) of the sequence $\left\{x_{n}\right\}$ defined by $x_{0} \in E$ and $x_{n+1}=T x_{n}+f$ for each $n=0,1,2, \ldots$, under the hypothesis that $\lim _{n \rightarrow \infty} T^{n} x$ exists for each $x \in E$. Kwon and Redheffer [4] considered equation (1) and the same sequence $\left\{x_{n}\right\}$ under the more general assumption that $\lim _{n \rightarrow \infty} T^{n} x$ exists for each $x$ in some subset of $E$. It is the purpose of this note to obtain generalizations of results in [4] by introducing a more general sequence $\left\{x_{n}\right\}$ (to be defined below) and obtaining results concerning $\left\{x_{n}\right\}$ analogous to those in [4].

Let $\left\{\lambda_{n}\right\}_{n=1}^{\infty}$ be a sequence of real numbers such that $0 \leqslant \lambda_{n} \leqslant 1$ for each $n=1,2, \ldots$, and $\lim _{n \rightarrow \infty} \lambda_{n}=0$. For notational simplicity we take $\lambda_{1}=1$. Define the infinite lower triangular matrix $A=\left[a_{n j}\right]$ by $a_{11}=\lambda_{1}=1, a_{i j}=0$ for each $j=2,3, \ldots, a_{n+1,1}=\lambda_{n+1}$ for each $n=1,2, \ldots, a_{n+1, j+1}$ $=a_{n j}\left(1-a_{n+1,1}\right)$ for each $n=1,2, \ldots$, and each $j=1,2, \ldots, n$, and $a_{n+1, j}=0$ for each $n=1,2, \ldots$, and each $j=n+2, n+3, \ldots$

REMARK 1 . We note that $A=\left[a_{n j}\right]$ satisfies

(M1) $a_{n j} \geqslant 0$ for every $n$ and $j$, and $=0$ for $j>n$,

(M2) $\sum_{j=1}^{n} a_{n j}=1$ for every $n$, and

(M3) $\lim _{n \rightarrow \infty} a_{n j}=0$ for every $j$.

Hence $A$ is a regular matrix (cf. [3, p. 87]). Suppose $x_{0} \in E$ and let the sequence $\left\{x_{n}\right\}$ be defined recursively by

$$
x_{n+1}=\left(1-\lambda_{n+1}\right)\left(T x_{n}+f\right)+\lambda_{n+1} x_{0}
$$

for each $n=0,1,2, \ldots$ This is a nonnormal Mann process which has been studied by Mann and Dotson.

Received by the editors December 16, 1974 and, in revised form, March 26, 1975.

AMS (MOS) subject classifications (1970). Primary 47 A50.

Key words and phrases. Iteration, linear equations. 
REMARK 2. If we take $\lambda_{n}=0$ for every $n$ then (2) yields the iterative process considered in [1] and [4] and the matrix $A$ reduces to the infinite identity matrix. If we take $\lambda_{n}=1 / n$ for every $n$ and $x_{0}=T y_{0}+f$ for $y_{0} \in E$ then (2) yields the iterative process studied by deFigueiredo and Karlovitz [2]. It is well known (by [2]) that this process of ten converges when the process in [1] and [4] does not.

Define the affine operator $S$ from $E$ to $E$ by $S x=T x+f$ for every $x \in E$. It is easily seen that

$$
S^{j} x=T^{j} x+\sum_{i=1}^{j} T^{i-1} f
$$

for every $x \in E$ and for every $j=1,2, \ldots$ Moreover

$$
x_{n+1}=\left(1-\lambda_{n+1}\right) S x_{n}+\lambda_{n+1} x_{0}
$$

for each $n=0,1,2, \ldots$ For each positive integer $n$, define polynomials $a_{n}(t)$ and $b_{n}(t)$ by

$$
a_{n}(t)=\sum_{j=1}^{n} a_{n j} t^{j-1}, \quad b_{n}(t)=\frac{1-a_{n}(t)}{1-t} .
$$

Condition (M2) of Remark 1 insures that $b_{n}(t)$ is a polynomial. Using (5) together with condition (M2) we have

$$
b_{n}(t)=\sum_{j=1}^{n} \frac{a_{n j}\left(1-t^{j-1}\right)}{1-t}=\sum_{j=1}^{n} a_{n j} \sum_{i=1}^{j-1} t^{i-1} .
$$

(We define $\sum_{i=1}^{0}=0$.) Now define linear operators $A_{n}$ and $B_{n}$ from $E$ to $E$ by $A_{n}=a_{n}(T)$ and $B_{n}=b_{n}(T)$ for each $n=1,2, \ldots$ By (5) we have

$$
(I-T) B_{n}=B_{n}(I-T)=I-A_{n}
$$

(where $I$ denotes the identity operator on $E$ ).

LEMMA 1. For each $n=1,2, \cdots$,

$$
x_{n}=\sum_{j=1}^{n} a_{n j} S^{j-1} x_{0} .
$$

Proof. The proof is by induction.

$$
\begin{aligned}
& x_{1}=\left(1-\lambda_{1}\right) S x_{0}+\lambda_{1} x_{0}=(1-1) S x_{0}+1\left(x_{0}\right)=x_{0}, \\
& x_{2}=\left(1-\lambda_{2}\right) S x_{1}+\lambda_{2} x_{0}=\left(1-\lambda_{2}\right) S x_{0}+\lambda_{2} x_{0} .
\end{aligned}
$$

If $x_{n}=\sum_{j=1}^{n} a_{n j} S^{j-1} x_{0}$ then

$$
x_{n+1}=\left(1-\lambda_{n+1}\right) S x_{n}+\lambda_{n+1} x_{0}=\left(1-\lambda_{n+1}\right) \sum_{j=1}^{n} a_{n j} S^{j} x_{0}+\lambda_{n+1} x_{0},
$$

where we have used that $S$ is an affine operator. But since $\lambda_{n+1}=a_{n+1,1}$ and $\left(1-a_{n+1,1}\right) a_{n j}=a_{n+1, j+1}$ for each $j=1, \ldots, n$, equation (8) gives 


$$
x_{n+1}=\sum_{j=1}^{n} a_{n+1, j+1} S^{j} x_{0}+a_{n+1,1} x_{0}=\sum_{j=1}^{n+1} a_{n+1, j} S^{j-1} x_{0} \text {. }
$$

This completes the proof of Lemma 1. Combining equations (3) and (7) we obtain

LEMMA 2. For every positive integer $n$,

$$
x_{n}=A_{n} x_{0}+B_{n} f
$$

Following [4] we define

$$
Q x=\lim _{n \rightarrow \infty} A_{n} x,
$$

whenever this limit exists (unless otherwise stated all statements about limits refer to convergence in the norm). We also define

$$
D(Q)=\left\{x \in E: \lim _{n \rightarrow \infty} A_{n} x \text { exists }\right\}, \quad N(Q)=\left\{x \in E: \lim _{n \rightarrow \infty} A_{n} x=0\right\} .
$$

It is clear that $D(Q)$ is a linear subspace of $E$ and that the operator $Q$ is linear on $D(Q)$.

REMARK 3. If $\lim _{n \rightarrow \infty} T^{n} x$ exists then, since $A$ is a regular matrix, $\lim _{n \rightarrow \infty} A_{n} x$ exists. We observe that in case $A$ is the Cesàro matrix $\left(\lambda_{n}=1 / n\right)$ various mean ergodic theorems give conditions under which $x \in D(Q)$.

Lemma 3. If $x \in D(Q)$ then $T x \in D(Q)$ and $Q(T x)=Q x$.

Proof. For any $n$ we have

$$
\begin{aligned}
A_{n}(T x) & =\sum_{j=1}^{n} a_{n j} T^{j} x \\
& =\sum_{j=1}^{n+1} a_{n+1, j} T^{j-1} x-a_{n+1,1} x+\sum_{j=1}^{n}\left(a_{n j}-a_{n+1, j+1}\right) T^{j} x .
\end{aligned}
$$

Using the property $a_{n+1, j+1}=a_{n j}\left(1-a_{n+1,1}\right)$ of $A$ and the linearity of $T$, we get

$$
\begin{aligned}
A_{n}(T x) & =\sum_{j=1}^{n+1} a_{n+1, j} T^{j-1} x-a_{n+1,1} x+a_{n+1,1} \sum_{j=1}^{n} a_{n j} T^{j} x \\
& =\sum_{j=1}^{n+1} a_{n+1, j} T^{j-1} x-a_{n+1,1} x+a_{n+1,1} T\left(\sum_{j=1}^{n} a_{n j} T^{j-1} x\right) \\
& =A_{n+1} x-a_{n+1,1} x+a_{n+1,1} T\left(A_{n} x\right) .
\end{aligned}
$$

But $\lim _{n \rightarrow \infty} a_{n+1,1}=0$ and $\lim _{n \rightarrow \infty} A_{n+1} x=Q x$ since $x \in D(Q)$. Moreover $T$ is continuous so that $\lim _{n \rightarrow \infty} T\left(A_{n} x\right)=T Q x$. Thus the right-hand side of equation (9) converges to $Q x$ as $n \rightarrow \infty$. Hence we conclude that $\lim _{n \rightarrow \infty} A_{n}(T x)=Q x$. Therefore $T x \in D(Q)$ and $Q(T x)=Q x$ so the lemma is proved.

REMARK 4. By Lemma 3, $x \in D(Q)$ implies 


$$
\lim _{n \rightarrow \infty} A_{n} T x=Q x=\lim _{n \rightarrow \infty} A_{n} x
$$

so that

$$
\lim _{n \rightarrow \infty}\left(A_{n} T x-A_{n} x\right)=0
$$

for every $x \in D(Q)$. By Lemma 3 , it is clear that $x \in D(Q)$ implies $T^{j} x \in D(Q)$ and $Q T^{j} x=Q x$ for every positive integer $j$. In addition $T$ commutes with $A_{n}$ for every $n$ and $T$ is continuous. Thus for $x \in D(Q)$,

$$
T(Q x)=Q(T x)=Q x .
$$

This is easily extended to $T^{j} Q x=Q x$ for each positive integer $j$.

Theorem 1. Suppose there exists $y \in E$ such that $y-T y=f$. Then $\left\{x_{n}\right\}$ converges if and only if $x_{0}-y \in D(Q)$.

Proof. From Lemma 2

$$
x_{n}=A_{n} x_{0}+B_{n} f
$$

for each $n=1,2, \ldots$ But $y-T y=f$ implies that

$$
B_{n} f=B_{n}(I-T) y \text { for each } n=1,2, \ldots
$$

Thus by equation (6)

$$
B_{n} f=y-A_{n} y \quad \text { for each } n=1,2, \ldots
$$

Therefore (11) yields

$$
x_{n}=A_{n}\left(x_{0}-y\right)+y \text { for each positive integer } n .
$$

Clearly $\left\{x_{n}\right\}$ has a limit if and only if $\left\{A_{n}\left(x_{0}-y\right)\right\}$ converges, i.e., if and only if $x_{0}-y \in D(Q)$.

This obviously generalizes Remark 1 of [4]. Before leaving Theorem 1, we note that if $\left\{x_{n}\right\}$ converges then the limit point is a solution of equation (1).

If $x \in E$ then $x$ is said to be an approximate solution of $u-T u=f$ provided that $x-T x-f \in N(Q)$. In the remainder of this note, $\rightarrow$ will denote convergence with respect to the weak topology on $E$.

THEOREM 2. If $x_{0}$ is an approximate solution of $u-T u=f$, then every weak subsequential limit point $x$ of $\left\{x_{n}\right\}$ satisfies $x-T x=f$.

Proof. Let $x$ be a weak subsequential limit point of $\left\{x_{n}\right\}$. Then there is a subsequence $x_{n_{j}}=A_{n_{j}} x_{0}+B_{n_{j}} f$ such that $x_{n_{j}} \rightarrow x$.

Now $I-T$ is (strongly) continuous and linear and hence $I-T$ is weakly continuous. Thus

$$
(I-T) x_{n_{j}} \rightarrow x-T x
$$

But

$$
(I-T) x_{n_{j}}=(I-T) A_{n_{j}} x_{0}+(I-T) B_{n_{j}} f
$$

for each $j$. 
Combining (12) and (13) we obtain

$$
A_{n_{j}} x_{0}-T A_{n_{j}} x_{0}+(I-T) B_{n_{j}} f \rightarrow x-T x .
$$

By (6) (and the fact that $T$ commutes with $A_{n_{j}}$ ) this becomes

$$
A_{n_{j}}\left(x_{0}-T x_{0}-f\right)+f \rightarrow x-T x .
$$

Hence

$$
A_{n_{j}}\left(x_{0}-T x_{0}-f\right) \rightarrow x-T x-f .
$$

But $x_{0}-T x_{0}-f \in N(Q)$ so that

$$
A_{n_{j}}\left(x_{0}-T x_{0}-f\right) \rightarrow 0 .
$$

The conclusion of the theorem follows immediately, since the weak topology is Hausdorff.

Theorem 2 clearly generalizes Remark 2 of [4].

Before proceeding to the next theorem we will need the following lemma, the proof of which is straightforward.

LeMma 4. If $x \in D(Q)$, then for each $n$,

(1) $A_{n} x, B_{n} x \in D(Q)$,

(2) $Q A_{n} x=Q x$, and

(3) $Q B_{n} x=\phi(n) Q x$ where

$$
\phi(n)=\left(\sum_{j=1}^{n} j a_{n j}\right)-1 \text { and } \lim _{n \rightarrow \infty} \phi(n)=+\infty .
$$

THEOREM 3. Suppose that $x_{0}, f \in D(Q)$ and that 0 is a weak subsequential limit point of $\left\{Q x_{n} / \phi(n)\right\}$. Then $x_{0}-T x_{0}-f \in N(Q)$.

Proof. We first note that $x_{n} \in D(Q)$ since by Lemma $4, A_{n} x_{0}, B_{n} f$ $\in D(Q)$ and $D(Q)$ is a linear subspace of $E$. By hypothesis there is a subsequence $\left\{Q x_{n_{j}} / \phi\left(n_{j}\right)\right\}$ such that

$$
Q x_{n_{j}} / \phi\left(n_{j}\right) \rightarrow 0 \text { as } j \rightarrow \infty .
$$

For each $j, x_{n_{j}}=A_{n_{j}} x_{0}+B_{n_{j}} f$. Since $Q$ is linear on $D(Q)$ we have

$$
Q x_{n_{j}} / \phi\left(n_{j}\right)=Q A_{n_{j}} x_{0} / \phi\left(n_{j}\right)+Q B_{n_{j}} f / \phi\left(n_{j}\right) .
$$

Using (2) and (3) of Lemma 4 we get

$$
Q x_{n_{j}} / \phi\left(n_{j}\right)=Q x_{0} / \phi\left(n_{j}\right)+\phi\left(n_{j}\right) Q f / \phi\left(n_{j}\right) .
$$

Therefore

$$
Q x_{n_{j}} / \phi\left(n_{j}\right)=Q x_{0} / \phi\left(n_{j}\right)+Q f .
$$

Since $\lim _{j \rightarrow \infty} \phi\left(n_{j}\right)=+\infty,\left\{Q x_{n_{j}} / \phi\left(n_{j}\right)\right\}$ converges (strongly) to $Q f$. Hence $\left\{Q x_{n_{j}} / \phi\left(n_{j}\right)\right\}$ converges weakly to $Q f$ and since the weak topology on $E$ is 
Hausdorff we conclude that $Q f=0$. Thus

$$
\lim _{n \rightarrow \infty} A_{n}\left(x_{0}-T x_{0}-f\right)=\lim _{n \rightarrow \infty}\left(A_{n} x_{0}-A_{n} T x_{0}\right) .
$$

From equation (10)

$$
\lim _{n \rightarrow \infty}\left(A_{n} x_{0}-A_{n} T x_{0}\right)=0
$$

Therefore

$$
\lim _{n \rightarrow \infty} A_{n}\left(x_{0}-T x_{0}-f\right)=0,
$$

i.e., $x_{0}-T x_{0}-f \in N(Q)$.

Theorem 3 contains Remark 3 of [4] as a special case.

Theorem 4. Suppose there exists $u \in D(Q)$ such that $u-T u=f$. Then

(a) every solution of $(1)$ is in $D(Q)$,

(b) $f \in N(Q)$, and

(c) $\left\{x_{n}\right\}$ converges if and only if $x_{0} \in D(Q)$. then

Proof. Suppose $y \in E$ and $y-T y=f$. Since $u \in D(Q)$ and $u-T u=f$

$$
B_{n} f=B_{n}(I-T) u=\left(I-A_{n}\right) u=u-A_{n} u
$$

where we have used equation (6). But $u \in D(Q)$ implies that $\lim _{n \rightarrow \infty} A_{n} u$ $=Q u$. Therefore

$$
\lim _{n \rightarrow \infty} B_{n} f=u-Q u \text {. }
$$

Now $y-T y=f$ and $y-y=0 \in D(Q)$ so that by Theorem 1 , the sequence $z_{n}=A_{n} y+B_{n} f$ converges (strongly) to some $z \in E$, i.e.,

$$
\lim _{n \rightarrow \infty}\left(A_{n} y+B_{n} f\right)=z
$$

Equations (14) and (15) combine to imply that

$$
\lim _{n \rightarrow \infty} A_{n} y=z-u+Q u \text {. }
$$

Therefore $y \in D(Q)$.

(b) For each $n, A_{n} f=A_{n} u-A_{n} T u$ but $u \in D(Q)$ so by equation (10)

$$
\lim _{n \rightarrow \infty}\left(A_{n} u-A_{n} T u\right)=0 .
$$

Hence $\lim _{n \rightarrow \infty} A_{n} f=0$, i.e., $f \in N(Q)$.

(c) Since $u-T u=f$ we have by Theorem 1 that $\left\{x_{n}\right\}$ converges if and only if $x_{0}-u \in D(Q)$. But since $u \in D(Q), x_{0}-u \in D(Q)$ if and only if $x_{0}$ $\in D(Q)$. Theorem 4 clearly generalizes Remark 4 of [4].

LEMMA 5. If $\left\{B_{n} f\right\}$ converges then

$$
\lim _{n \rightarrow \infty}\left(B_{n+1} f-T B_{n} f\right)=f
$$


Proof. For every $n$ we have

$$
\begin{aligned}
B_{n+1} f & =\sum_{j=2}^{n+1} a_{n+1, j} \sum_{i=1}^{j-1} T^{i-1} f\left(\text { recall that } \sum_{i=1}^{0}=0\right), \\
T B_{n} f & =\sum_{j=2}^{n} a_{n j} \sum_{i=1}^{j-1} T^{i} f .
\end{aligned}
$$

Subtraction yields

$$
B_{n+1} f-T B_{n} f=\left(\sum_{j=2}^{n+1} a_{n+1, j}\right) f+\sum_{j=2}^{n}\left(a_{n+1, j+1}-a_{n j}\right) \sum_{i=1}^{j-1} T^{i} f .
$$

By property (M2) of the matrix $A$ and the condition $a_{n+1, j+1}=a_{n j}\left(1-a_{n+1,1}\right)$ we have

$$
\begin{aligned}
B_{n+1} f-T B_{n} f & =\left(1-a_{n+1,1}\right) f-\sum_{j=2}^{n} a_{n+1,1} a_{n j} \sum_{i=1}^{j-1} T^{i} f \\
& =\left(1-a_{n+1,1}\right) f-a_{n+1,1} T B_{n} f .
\end{aligned}
$$

If $\left\{B_{n} f\right\}$ converges to $u$ then by the continuity of $T$,

$$
\lim _{n \rightarrow \infty} T B_{n} f=T u
$$

Therefore

$$
\lim _{n \rightarrow \infty}\left[\left(1-a_{n+1,1}\right) f-a_{n+1,1} T B_{n} f\right]=f,
$$

since $\lim _{n \rightarrow \infty} a_{n+1,1}=0$. The conclusion of the lemma follows immediately.

THEOREM 5. $\left\{B_{n} f\right\}$ converges if and only if there exists $u \in D(Q)$ such that $u-T u=f$.

Proof. If there exists $u \in D(Q)$ such that $u-T u=f$ then by equation (6)

$$
B_{n} f=B_{n}(I-T) u=\left(I-A_{n}\right) u=u-A_{n} u .
$$

Since $u \in D(Q)$, it is clear that $\left\{B_{n} f\right\}$ converges.

Conversely if $\left\{B_{n} f\right\}$ converges to $u$ then $\left\{T B_{n} f\right\}$ converges to $T u$. Therefore $\left\{B_{n+1} f-T B_{n} f\right\}$ converges to $u-T u$. But by Lemma $5,\left\{B_{n+1} f-T B_{n} f\right\}$ converges to $f$. Hence $u-T u=f$. It remains only to show that $u \in D(Q)$. Clearly

$$
\lim _{n \rightarrow \infty}\left(A_{n}(0)+B_{n} f\right)=u
$$

so by Theorem $1,-u=0-u \in D(Q)$. Since $D(Q)$ is a linear subspace of $E$, it is clear that $u \in D(Q)$.

As a special case Theorem 5 contains Remark 5 of [4], since if $A=\left[a_{n j}\right]$ is the infinite identity matrix then

$$
b_{n}(t)=1-t^{n-1} / 1-t=1+t+\cdots+t^{n-2}
$$

and so $\left\{B_{n} f\right\}=\left\{f+T f+\cdots+T^{n-2} f\right\}$. 


\section{REFERENCES}

1. F. E. Browder and W. V. Petryshyn, The solution by iteration of linear functional equations in Banach spaces, Bull. Amer. Math. Soc. 72 (1966), 566-570. MR 32 \#8155a.

2. D. G. deFigueiredo and L. A. Karlovitz, On the approximate solution of linear functional equations in Banach spaces, J. Math. Anal. Appl. 24(1968). 654-664. MR 38 \#6415.

3. C. Goffman and G. Pedrick, First course in functional analysis, Prentice-Hall, Englewood Cliffs, N. J., 1965. MR 32 \# 1540.

4. Y. K. Kwon and R. M. Redheffer, Remarks on linear equations in Banach space, Arch. Rational Mech. Anal. 32 (1969), 247-254, MR 40 \#6284.

Department of Mathematics, North Carolina State University, Raleigh, North CaroLINA 27607 